\title{
Elementary School Childrens Development Viewed from The Children's Health Perspective: An Integrative Review
}

\section{Pearl Ed G. Cuevas}

Centro Escolar University, Manila, Philippines

$\triangle$ pearled.cuevas@gmail.com

\begin{abstract}
This study aimed to ascertain the development of elementary school children in the digital age from their health perspective. This integrative review is aimed at creating a health program for elementary school children in order to guide health workers in creating meaningful, focused, and structured activities that will address their health needs in the digital age. The integrative review as a methodology provides a synthesis of knowledge and the applicability of results of significant studies into practice. Its objective is to present the phases of children's health in an integrative review so that relevant aspects of the literatures will be taken into account when using this methodological resource. As the influence of digital technology-- and especially the internet-has increased, the debate about its effect on elementary school children's development has grown louder. We wonder, is the internet a threat to their over-all health and well-being? Digital dependency has shown to have a possible impact on brain development and cognition of elementary school children. In conclusion, the digital technology has already changed the world-and as more and more children go online around the world, it is increasingly changing childhood (UNICEF, 2017).
\end{abstract}

Keywords: Elementary school children's development, children's health, integrative review.

How to Cite: Cuevas, P. E. (2018). Elementary School Childrens Development Viewed from The Children's Health Perspective: An Integrative Review. Mimbar Sekolah Dasar, 5(3), 131-137. doi:http://dx.doi.org/10.17509/mimbar-sd.v5i3.14498.

INTRODUCTION This study aimed to ascertain the development of elementary school children in the digital age from their health perspective. This integrative review is creating a health advocacy for elementary school children in order to guide health workers in creating meaningful, focused, and structured interventions that will address their health needs in the digital age.

The earliest years of our lives set us on the path leading toward-or away fromgood health. There is strong evidence that demonstrates the effects of early childhood interventions, especially for children with the greatest social and economic advantage, to good health. Therefore, families of all socio-economic levels must experience the benefits from early childhood programs that translate into improved development and health. It is because improving early childhood social circumstances in this digital age is one of the most effective ways for a society to achieve its health potential (Braveman \& Egerter, 2008).

\section{METHODOLOGY}

The integrative review as a methodology provides a synthesis of knowledge and the applicability of results of significant studies 
Pearl Ed G. Cuevas, Elementary School Childrens Development Viewed...

into practice. Its objective is to present the phases of children's health in an integrative review so that relevant aspects of the literatures will be taken into account when using this methodological resource. These include quantitative and qualitative literatures within the past five years that is related to the key variables in the study. A total of 30 studies was reviewed and synthesized to get the final results for analysis.

\section{RESULTS}

As the influence of digital technology-and especially the internet-has increased, the debate about its effect on elementary school children's development has grown louder. We wonder, is the internet a threat to their over-all health and well-being? Digital dependency has shown to have a possible impact on brain development and cognition of elementary school children. Their voices matter ever more-and louder than ever before-in a digital world. A world they are not only inheriting, but helping to shape (UNICEF, 2017).

Several studies show evidence that children's social and economic condition in this digital age have direct effects on health and development. It is widely recognized that factors such as nutrition, housing quality, and household and community safety-all linked with family resources-are strongly linked with child health. Research shows that children's nutrition varies with parents' income and education and can have lasting effects on health throughout life. There are also results that consistently link children's development with social and economic advantages and disadvantages in the home environments of young children. Compared to children in the highestincome families, children in the lowestincome families were least likely to have the needed skills, but children in middleclass families also performed less well, both socially and academically, than those at the top. The links between children's development and adult health may involve "connecting the dots" through effects on important social outcomes. There is very strong evidence that social disadvantages experienced in childhood, like no access to the internet, can limit children's opportunities for health throughout life (Braveman \& Egerter, 2008).

\section{The Evidence Linking Early Childhood Experiences With Health}

Studies of early childhood experiences and its links with health: research findings have consistently shown that (a) social experiences in early childhood are linked to brain, cognitive and behavioral development; and (b) brain, cognitive and behavioral development are in turn strongly linked-often through effects of educational attainment-to an array of important health outcomes.

Studies of early child development programs: findings from observational and experimental studies provide evidence of direct links between particular early child development program and important 
health and health outcomes. Other experimental and observational studies indirectly link a particular early childhood development interventions with health outcomes by demonstrating their impact on social outcomes that have wellestablished and important health consequences.

However, the internet of things, artificial intelligence and machine learning are creating new opportunities as well as new challenges. In UNICEF's (2017) state of the World's Children- Children in a Digital World, it has stated some key messages about the development of elementary school children in the digital age.

Digital technology has already changed the world- and as more and more children go online around the world, it is increasingly changing childhood. Smartphones are fuelling a "bedroom culture" with online access for many children becoming more personal, more private and less supervised.

Connectivity can be a game changer for some of the world's most marginalized children, helping them fulfill their potential and break intergenerational cycles of poverty. Digital technologies are bringing opportunities for learning and education to children, especially in remote regions and during humanitarian crises.

Digital access is becoming the new dividing line, as millions of the children who could most benefit from digital technology are missing out. Digital divides also mirror prevailing economic gaps, amplifying the advantages of children from wealthier backgrounds and failing to deliver opportunities to the poorest and most disadvantaged children.

Digital technology can also make children more susceptible to harm both online and off. Already vulnerable children may be at greater risk of harm, including loss of privacy. While attitudes vary by culture, children often turn first to their peers when they experience the risks and harm online, making it harder for parents to protect them.

The potential impact of Internet Computer Technology's on children's health and happiness is a matter of growing public concern-and an area that is ripe for future research and data. Although most children who are online view it as a positive experience, many parents and teachers worry that immersion in screens is making children depressed, creating internet dependency and even contributing to obesity.

Indeed digitalization is changing childhood, for better or for worse. The internet has changed how children form and maintain their friendships, allowing them to maintain almost-constant contact with peers. They have also transformed how children spend their leisure time, providing them with a constant feed of videos, social media updates and highly immersive games. But many adults fear these changes are not all for the better, 
Pearl Ed G. Cuevas, Elementary School Childrens Development Viewed...

and worry that excessive screen time is isolating children from their families and surroundings, fuelling depression and even making children obese.

\section{ANALYSIS}

The internet reflect and amplify the best as well as the worst of human nature. It is a tool that will always be used for good and for ill. Our job is to mitigate the harms and expand the opportunities digital technology makes possible (UNICEF, 2017). Technology needs to be supported by strong teachers, motivated learners and sound pedagogy. Highly trained and responsive caregivers, small class sizes with low child-teacher ratios, safe and adequate physical environments, and age -appropriate activities focused on enhancing the cognitive and socialemotional development of the child is often cited as hallmarks of high quality child development.

The early elementary school-age child 16 to 8 years old) has moved from being a preschooler closely tied to family to the expanded world of middle childhood. There are three great "outward journeys" of middle childhood -Social, Physical \& Mental. However, as children grow, the capacity of digitalization to shape their life experiences grows with them, offering seemingly limitless opportunities to learn and socialize, to be counted and be heard. Digital technology and interactivity also pose significant risks to children's safety, privacy and well-being, magnifying threats and harms that many children face offline and making already vulnerable children even more vulnerable.

Social and emotional development is ensued when a child has attachment to friends. The "Real" friends and not merely virtual friends from the internet. Elementary children must experience having a "best friend", an "enemy" and even how to play with the same sex. However, at the present time, the internet has made it easier for children to connect to one another and share experiences online. It has also made it easier to use those new channels of connectivity and communication for online bullying, with a much greater risk-and thus potentially greater risk-than off-line bullying. When children play with both sexes in a virtual world, they are not cognizant of their sex differences, but look at each other as "all children". Thus, looking through equal lenses, all children can receive good feedback and/or harsh comments.

During this age, children also have the strong desire for affection and attention of parents. Thus, parents must make sure that it is not the internet that is available for the children when they need answers to questions about life. When failures arise, concentrate on children's successes and teach them how to learn from criticism. They may try out new behaviors that they get from the internet, but make sure that it is not dangerous and open your lines of communication to ask if the child has learned anything from it. There will be times when even their patience will be 
tested, perhaps in use internet games. Parents need to remember that the children's conscience is being formed at this stage and some children in this age group release tension through physical activity. So parents must have an open space to do physical activity, even at home.

At this stage, children's need for food may also fluctuate with activity. Though in the digital age, daily activity consist mostly of playing video games. On the positive side, video games may have beneficial effects on children's visual acuity and ability to learn. However, those who use the computer for longer periods of time tend to be malnourished-either undernourished or obese. Taking a 'Goldilocks' approach to children's screen time-not too much, not too little-and focusing more on what children are doing online, can better protect them and help them make the most of their time online. Even as the internet and digital entertainment have spurred tremendous creativity and expanded children's access to a wealth of enriching and entertaining content, a question is also raised related to digital dependency or screen addiction among children. And even such technologies have enlarged platforms for the free expression of ideas, broadened distribution of hate speech and other negative content that can shape the children's view of the world and even of themselves.
Children in this age group begin to form ideas mentally. Children who have been introduced to the computer early on are expected to be more advance in this development. There are computer games that help them even in the next level of mental development, such as sequencing and ordering, preparing the way for math skills. During this stage, children form a more advanced understanding of numbers as the computer has already introduced this to them early on. Elementary children also tend to talk as they learn, and they learn better if active while learning. This is perhaps the best contribution of the computer that helps our children become multi-taskers. Normally, their interest span is short, for about 20 minutes, unless when they are engrossed in the computer.

However, there is a clear gap in children's knowledge about risks online, and despite rapidly increasing usage among elementary children, many lack digital skills and the critical ability to gauge the safety and credibility of content and relationships they experience online. This reflects a need for a more widespread digital literacy opportunity that can both safeguard and empower children.

Gaps in our knowledge about children's lives online, including the impact of connectivity on a range of areas, such as cognition, learning and social emotional development, make it more difficult to develop dynamic policies that get ahead of issues by addressing risks and making 
Pearl Ed G. Cuevas, Elementary School Childrens Development Viewed...

the most of opportunities. Gaps in our understanding of how children feel about their experience of connectivity-including their perception of risks-further limit us.

One thing is certain, by protecting children from the worst digital technology has to offer, and expanding their access to the best, we can tip the balance for the better (Lake in UNICEF 2017).

\section{DISCUSSIONS}

In conclusion, the digital technology has already changed the world-and as more and more children go online around the world, it is increasingly changing childhood (UNICEF, 2017). Schools should provide children with the basic rules and awareness of internet use and abuse, as part of elementary education. Many studies support the conclusion that children who participate in high quality early childhood development programs experience a range of immediate and long-term health benefits (Braveman \& Egerter, 2008).

The integrative literature review showed an evaluation on the strength of scientific evidence on the development of elementary school children and the effect of the digital age into the children's health. Technology and internet companies should take steps to prevent their networks and services from being used by offenders to collect and distribute child sexual abuse images or commit other violations against children. Media stories about the potential impact of connectivity on children's healthy development and well-being should be grounded in empirical research and data analysis.

To ensure social and emotional development, parents must give children lots of positive feedback for good behavior and let the child help define rules. This will build self-esteem and cut down negative behaviors. Since this is the age of being competitive, encourage non- competitive games and help your child set individual goals. There must also be open "reporting" of activities to family. Parents need to set aside time specifically to listen and talk to children with no interruptions. It is important that parents help the child develop problem-solving skills. Encourage children to ask the wisdom of elders when they have questions about life and not merely to google the answer. Adults need to give children measured doses of realistic information to help them handle their fears. Remember that a positive selfconcept continues to develop with successful experiences. Communicate with children about why self-control is important and why they should learn to be patient, share and respect the rights of others.

Physically, muscle coordination and control is uneven and incomplete. Parents need to encourage the child to participate in activities using high energy. Children of this age need 10-12 hours of sleep each night. For children who learned computer use early, parents may 
encourage them to work briefly with small motor tasks, then switch to running and jumping as this is important in physical development. At this stage, children begin to learn the value of "work". They need regular, realistic chores at home and school. As they now begin to understand the value and uses of money.

A Nurse-family partnership and parents as teacher's activity is recommended to provide parent training and supportive guidance with the goal of increasing parents' self-efficacy and life skills. Children who participate in early child development programs are more likely to be healthy, have higher earnings as adults, and are less likely to commit crimes and receive public assistance. These benefits translate into tremendous savings for society (Braveman \& Egerter, 2008). This study hopes to help guide more effective policymaking and more responsible parental practices to benefit children in a digital age. This current knowledge tells us that investing in improving our children's development in this digital age is probably the most effective strategy in realizing their health potential for the future.

\section{CONCLUSION}

In conclusion, the digital technology has already changed the world-and as more and more children go online around the world, it is increasingly changing childhood (UNICEF, 2017). The integrative literature review showed an evaluation on the strength of scientific evidence on the development of elementary school children and the effect of the digital age into the children's social, physical and mental health.

\section{REFERENCES}

Braveman, P., \& Egerter, S. (2008). Overcoming obstacles to health: report from the Robert Wood Johnson Foundation to the Commission to Build a Healthier America: Robert Wood Johnson Foundation.

UNICEF. (2017). The State of the World's Children 2017-Children in a Digital World, December 2017, 14. 